\title{
Arbeitsmarkt Leiharbeit - Risiken und Chancen der künftigen Dienstleistungsfreiheit
}

\section{DIE ZEITARBEITSBRANCHE VOR NEUEN HERAUSFORDERUNGEN}

Die Zeitarbeitsbranche durchlebt spannende Zeiten: Einerseits steigen aufgrund der anhaltenden Konjunkturbelebung die Zahlen der Leiharbeitnehmer kontinuierlich an. Nach Angaben des Instituts der deutschen Wirtschaft waren im Dezember 2010 ca. 921.000 Arbeitnehmer in Leiharbeit beschäftigt, ${ }^{1}$ was einem Anstieg von nahezu $40 \%$ innerhalb eines Jahres entspricht und den Verleihern Rekordgewinne beschert. Andererseits stehen 1.600 Verleiher, ${ }^{2}$ die ihre Beschäftigten nach den Tarifen der Tarifgemeinschaft Christlicher Gewerkschaften für Zeitarbeit und Personalserviceagenturen (CGZP) entlohnten, aufgrund möglicher Nachzahlungen vor dem Aus, da es dieser Gewerkschaft nach dem abschließenden Urteil des Bundesarbeitsgerichts ${ }^{3}$ an Tarifmächtigkeit fehlt. Die nächste Herausforderung für die Verleiher wird der 1. Mai 2011 sein. Ab dann gilt für die acht, der Europäischen Union 2004 beigetretenen Staaten Mittel-und Osteuropas (MOE-Staaten) die völlige Dienstleistungsfreiheit und Arbeitnehmerfreizügigkeit. Dies schürt die Befürchtung, dass damit die polnischen, tschechischen oder auch deutschen Verleiher mit Niederlassungen in diesen Ländern mit Tarifverträgen von unter $5 €$ auf den deutschen Markt drängen. Dabei herrscht schon heute in Deutschland unter den Leiharbeitsfirmen ein immenser Verdrängungswettbewerb, der sich nahezu ausschließlich über den Preis reguliert. Da ein Drittel der Leiharbeiter als „Helfer“, also in an- oder ungelernten Tätigkeiten beschäftigt ${ }^{4}$ und die Marge in diesem Bereich ohnehin gering ist $(0,45$ bis $1 €$ pro Stunde), würden die Preise völlig zusammenbrechen und Verleiher, die sich an die gültigen Tarifverträge und Gesetze halten, würden zugunsten der „Schwarzen Schafe“ und „Küchentischverleiher mit Laptop und Telefon“ besonders unter Druck kommen.

Doch viele deutsche Ent- und Verleiher scheinen „vorbereitet“ zu sein. Sie wollen die Möglichkeiten der Lohndifferenz nutzen und Einsparungen in Millionenhöhe erreichen: ${ }^{5}$ Anfragen deutscher Entleiher an Verleiher in den MOE-Staaten sind bereits erfolgt, um hiesige Leiharbeitnehmer durch polnische, tschechische oder ungarische auszutauschen. Andere haben sich an polnische Gewerkschaften gewandt, um mit ihnen Tarifverträge auszuhandeln und so billige Arbeitskräfte nach Deutschland vermitteln zu können. Und in Polen niedergelassene deutsche Verleiher haben Firmentarifverträge mit Stundenlöhne von 4 bis $5 €$ vorbereitet, die im ungünstigsten Falle nach dem 1. Mai 2011 rechtwirksam sein könnten. ${ }^{6}$

\section{DER TRECK NACH WESTEN}

Experten schätzen den Zuzug aus den MOE-Staaten auf mehrere 100.000 Leiharbeiter. Ob diese Zahlen auch nur annähernd zutreffen, ist allerdings fraglich. Denn zum einen wird die befürchtete Arbeitnehmermobilität gemeinhin überschätzt. So blieb beispielsweise trotz niedriger Löhne etwa in der Ostslowakei und in Ostungarn eine inner-nationale Massenwanderung in die prosperierenden Gebiete Westungarns und der Westslowakei aus. ${ }^{7}$ Zum Zweiten hängt der Wanderungssog auch vom Kosten-NutzenKalkül der potenziellen Leiharbeitnehmer ab. Dauerhaft lange Anfahrtszeiten sowie hohe Transport- und Unterkunftskosten müssen einkalkuliert werden. Schon heute verlangen Verleiher für Shuttle-Services und Sicherheitskleidung immense Gebühren und gewähren nur geringe Kostenzuschüsse für Fahrt und Unterkunft. Ebenso werden die osteuropäischen Leiharbeiter die erwartbaren Gehälter mit Blick auf mögliche Wechselkursschwankungen, Mehr- kosten der Lebenshaltung und/oder die erwartbare soziale Entwurzelung bewerten. Da zudem die florierenden Grenzregionen Polens und Tschechiens sogar teilweise unter einem Fachkräftemangel leiden und die ostdeutschen Grenzregionen aufgrund des niedrigen Lohnniveaus wenig attraktiv für die Leiharbeitsmigranten sind, wird bei der anstehenden Öffnung vermehrt mit dem Zuzug aus den mittleren und östlichen Landesteilen in die westdeutschen

1 IW-Nachrichten vom 15.12.2010: Zeitarbeit Arbeitsplätze in Gefahr, http://www.iwkoeln de/Publikationen/IWNachrichten/tabid/123/ articleid/30738/Default.aspx.

2 SüddeutscheZeitung-Newstickervom 14.12.2010: "Anwalt sieht Zeitarbeitsbranche in Gefahr" http://newsticker.sueddeutsche.de/list/id/ 1083213.

3 BAG-Urteil vom 14.12.2010: 1 ABR 19/10.

4 Bundesagentur für Arbeit (Hrsg.) (2010): Durchstarten - Zeitarbeit, Nürnberg, http://www.arbeitsagentur.de/zentraler-Content/ Veroeffentlichungen/Berufsorientierung/ Durchstarten-Zeitarbeit-Ausgabe-2010.pdf, S. 7

5 Report München vom 29. November 2010: "Niedriglohn Made in Germany. Die Zeitarbeitsbranche unter Druck", http://origin-www.bronline.de/das-erste/report-muenchen/reportmuenchen-lohndumping-ID1291030573389.xml.

6 Frankfurter Rundschau online, 2.11.2010: „Offener Arbeitsmarkt 2011 - Dumping-Verträge sind längst entworfen“", http://www.fr-online. de/wirtschaft/spezials/dumping-vertraege-sindlaengst-entworfen/-/1473644/4794812/-/index. html.

7 Agentur für Handelsförderung des Ministeriums für Industrie und Handel der Tschechischen Republik in Österreich, 30.12.2010: „Arbeitsmarktöffnung lässt Osteuropäer kalt“", http://www. czechtrade.at/neuheiten/arbeitsmarktoffnunglasst-osteuropaer-kalt-18179/.

Sandra Siebenhüter, Dr., Katholische Universität Eichstätt-Ingolstadt; Leiterin des Forschungsprojektes "Neue Spaltungslinien am Arbeitsmarkt - Migrantinnen und Migranten in Leiharbeit" der Otto BrennerStiftung. Arbeitsschwerpunkte: Leiharbeit, Management und Führung, Suchtprävention. e-mail: sandra.siebenhueter@ku-eichstaett.de 
Boom-Regionen gerechnet. Laut Umfrage der Warschauer Zeitung Gazeta Wyborcza vom September 2010 sind in Polen rund eine halbe Million Menschen bereit, für einen Job ins Nachbarland zu gehen. ${ }^{8}$

Vieles spricht dafür, dass vor allem Arbeitslose oder Geringqualifizierte den Weg nach Westen antreten. Letztere arbeiten in ihrer Heimat für Löhne von unter 1,50€ und würden hier besonders den Leiharbeitsmarkt im Hilfskräftebereich unter Druck bringen.

\section{DROHT EIN AUSTAUSCH DER LEIHARBEITNEHMER?}

Ob dieses Szenario eintritt, hängt von den Entleihern und den Verleihern, aber auch von den Leiharbeitskräften selbst ab.

Bereits unter den Entleihern zeigen sich Interessenunterschiede: Kleinere Entleiher bevorzugen regionale Leiharbeitnehmer, die aufgrund ihrer geringeren Mobilität besonders stark an einer Übernahme interessiert und im Sinne des Entleihers besonders motiviert und loyal sind. $\mathrm{Zu}$ dem nutzen Mittelständler Leiharbeit als Rekrutierungsbörse für neue Stammmitarbeiter und werden deswegen auch weiterhin regional verfügbare Leiharbeitnehmer präferieren. Der massenhafte Wechsel zu billigeren Verleihern ist somit nicht unbedingt vorprogrammiert. Auch streben gerade mittelständische Entleiher nach einer langfristigen, stabilen und berechenbaren Zusammenarbeit mit Verleihern. Sie wünschen sich feste Ansprechpartner und kurze Kommunikationswege. Zudem legen sie einen hohen Wert auf die Einhaltung von Arbeitssicherheitsstandards und erwarten engagierte, leistungsbereite, deutschsprachige und auch fachnahe Leiharbeitnehmer. Darüber hinaus bevorzugen sie die Zusammenarbeit mit etablierten und in der Region bekannten Verleihern, um so die Gefahr zu minimieren, dass ein Verleiher in Konkurs geht und auch an sie mögliche Regressansprüche für Sozialleistungen gestellt werden. Nicht zuletzt erkennen viele inzwischen auch den hohen Stellenwert eines guten Miteinanders von Stammbelegschaft und Leiharbeitnehmern und versuchen, Spannungen soweit als möglich zu vermeiden, indem sie Leiharbeitnehmer kaum auswechseln, um so Anlernzeiten und damit letztlich auch die Fehlerhäufigkeit zu reduzieren.

Anders einige große Industrieunternehmen, die Hunderte von Helfern in Leih- arbeit beschäftigen. Ihre Einkaufsabteilungen werden bemüht sein, die „Beschaffung“ von Leiharbeitskräften unter Nutzung der ab 1. Mai 2011 erweiterten Möglichkeiten zu optimieren. Zu ihrem Geschäftsgebaren zählt, dass Leiharbeitnehmern seitens des Entleihers „angeboten“ wird, zu einem kostengünstigeren Verleiher zu wechseln. ${ }^{9}$ Kostenreduzierung ist hier das oberste Ziel. Sie wird erreicht durch die Verminderung der Zahl der Verleiher, durch Master Vendor Verträge (ein Generalverleiher), Nutzung von Preisnachlässen bei Erreichen einer bestimmten Umsatzhöhe, durch langfristige „Abnahmegarantien“ und automatisierte Bestell-/Rechnungsprozesse mit dem Verleiher. Unter diesem Gesichtspunkt sind Niedrigtarife und osteuropäische Verleiher mit einem geringeren Overhead willkommene Möglichkeiten, Gewinnaufschläge zu begrenzen. Ebenso wird es für Entleiher dieses Typs leichter, bei Neu-Ausschreibungen polnische Verleiher, deutsche Verleiher mit Niederlassungen in Polen und Tschechien und nationale Verleiher in einen noch stärkeren Wettbewerb zu zwingen.

Der Preiswettbewerb hat allerdings auch für Verleiher seine Grenzen. Zum einen brauchen selbst sie eine tarifliche Untergrenze, um ihre Mehrkosten gegenüber dem Entleiher zu rechtfertigen. Für die osteuropäischen Verleiher stellt sich zudem die Frage, ob mittelfristig nicht Ost-Europa das größere Wachstumspotenzial verspricht als der deutsche gesättigte Markt. Denn auch die dortigen Unternehmen werden bei steigender Lohnspirale ihren politischen Druck verschärfen, um den dortigen Arbeitsmarkt zu deregulieren. Das bedeutet für die Verleiher höhere Gewinne bei gleichzeitig weniger arbeits-, vertrags- und steuerrechtlichem Aufwand. Und schließlich sind Leiharbeitnehmer nicht durchweg Arbeitskräfte ohne jedwede Marktmacht. Zuziehende Leiharbeiter aus den MOE-Staaten werden ihre Einkommenssituation mit den hiesigen Beschäftigten vergleichen und Möglichkeiten zu Verbesserungen ohne Zögern ergreifen. Denn jemand, der bereit ist, aus Kattowitz nach Deutschland zu kommen, um zu arbeiten, für den ist München, Wiesbaden oder Hannover nahezu gleich weit entfernt und seine Loyalität zu einem schlechten Verleiher wird sich in Grenzen halten.

Gleichwohl ist nicht auszuschließen, dass es gerade in grenznahen und boomenden Regionen zu einer Verschärfung im Helferbereich, d.h. zu einer Unterbie- tungskonkurrenz zwischen hiesigen und zuwandernden Leiharbeitskräften hinsichtlich der Akzeptanz schlechter Arbeitsbedingungen kommen wird. Dass hiesige Leiharbeitnehmer im Helferbereich trotz fehlender Arbeitsmarktmacht ggf. vor einem beliebigen Lohndumping "geschützt “ sind, hat eher makaber anmutende Gründe: Die allermeisten Leiharbeitnehmer im Helferbereich sind Arbeitslosengeld (ALG)-IIBezieher, also Aufstocker. Sie werden, wie wir aus unseren Interviews wissen, einen „400-€-Job mit Aufstockung“ einem Leiharbeitsverhältnis mit stets wechselnden zeitlichen und örtlichen Arbeitseinsätzen vorziehen. Andere weigern sich inzwischen, überhaupt noch in Leiharbeit zu arbeiten, da sie nur schlechte Erfahrungen mit Verleihern gemacht haben und immer nur tage- oder wochenweise beschäftigt werden, was nichts anderes heißt, als zwischen einem Leiharbeitsjob mit Aufstockung und Arbeitslosigkeit hin- und herzuwechseln. Die damit verbundenen psychischen Belastungen können sich die wenigsten von uns vorstellen. Leiharbeitnehmer mit einem solchen Erfahrungshintergrund wären eher bereit, in andere Formen der Prekarität wie Minijobs, (Schein-)Selbstständigkeit, Schwarzarbeit etc. zu flüchten, als eine weitere Verschärfung des Drucks in der Leiharbeit hinzunehmen. ${ }^{10}$

Im Blick $\mathrm{zu}$ behalten ist aber auch ein weiteres Szenario, das schon heute in deutschen Betrieben Wirklichkeit ist: der Austausch von Leiharbeitern gegen Werkvertrags/Dienstleistungsnehmer. Diese vertragliche Konstruktion bietet aktuell die Schlupflöcher, um Ansätzen zur Regulierung der Leiharbeit wie EqualPay-Vereinbarungen, einen branchenspezifischen Mindestlohn, aber auch die Konsultationsrechte der Interessenvertretung zu umgehen.

\section{DIE BEDROHUNG ALS POLITISCHEN AUFTRAG AUFGREIFEN!}

An dieser Entwicklung wird deutlich, dass wir es letztlich mit einer grundlegenden

\footnotetext{
8 Ebd.

9 Augsburger Allgemeine vom 6.9.2010: Neuer Chef, altes Leid, http://www.augsburgerallgemeine.de/Home/Lokales/Augsburg-Stadt/ Lokalnews/Artikel, - Neuer-Chef-altes-Leid-_ arid,2236917_regid,2_puid,2_pageid,4490.html.

10 Diese Einschätzungen haben wir aus einem empirischen Forschungsprojekt (vgl. hierzu die Autorenangaben) gewonnen.
} 
Krise der Arbeitsbedingungen zu tun haben. Die am 1. Mai dieses Jahres eintretende Dienstleistungsfreiheit und Arbeitnehmerfreizügigkeit sollte deswegen Anlass sein, die überfällige Debatte zu den arbeitsmarkt- und sozialpolitischen Bedingungen in unserem Land erneut anzustoßen. Denn die Missstände in der Leiharbeitsbranche dauern bereits seit Jahren an und der Wettbewerbsdruck wurde bisher einzig und allein auf dem Rücken der Leiharbeitnehmer ausgetragen. Ein großflächiges Stillhalten der Betroffenen aus Unwissenheit um ihre Rechte, aus purer Existenzangst oder aus falsch verstandenem Stolz, gepaart mit einem politischen Unwillen und einem Desinteresse seitens der Medien und der Forschung, ließ andere Akteure im Feld der Leiharbeit sehr mächtig werden. Sie haben die Agenda mit populistischen und in diesem Sinne mehrheitsfähigen Schlagworten geprägt: Mehr Wettbewerbsfähigkeit, mehr Flexibilität, weniger Arbeitslosigkeit durch Leiharbeit. Stets zu kurz gekommen ist dabei die soziale Realität der Leiharbeitnehmer: Probleme mit der Abrechnung, mit unzureichender Arbeitsausrüstung, die Willkür von Personaldisponenten, die Einsatzzeiten und Einsatzorte festlegen, selbstherrliche Vorarbeiter und Meister, die nach Nasenfaktor darüber entscheiden, ob eine Leiharbeitskraft morgen nochmals „arbeiten darf“. Die Konkurrenz unter den Leiharbeitern ist im Bereich der Hilfskräfte heute schon immens und wird nun nochmals verstärkt. Sie erleben tagtäglich, wie sie seitens der Stammbelegschaft als Lückenbüßer für ungeliebte Arbeiten missbraucht werden. Leiharbeitnehmer, die sich gegen diese Zustände wehren, sind die Ausnahme. Dass dies auch die Verleiher wissen und aus der Not der Leiharbeitnehmer ihre „Handlungsoptionen“ generieren, das ist der eigentliche Skandal.

Die erweiterte Freizügigkeit sollte aber auch Anlass sein, die Debatte um Leiharbeit endlich aus einer rein nationalen Wahrnehmungsperspektive zu lösen. Mehr noch: Die mit der Dienstleistungsfreiheit und Arbeitnehmerfreizügigkeit verbundene „Zunahme von Verflechtungen und
Interaktionen über nationale Grenzen hinweg, erzwingt die Neuvermessung sozialer Ungleichheit". ${ }^{11}$ Große Entleiher sind schon lange nicht mehr dem nationalen Rahmen verhaftet und werden dort ihre Arbeitskräfte rekrutieren, wo sie möglichst billig sind. Doch wenn ungelernte polnische Leiharbeiter auf ungelernte deutsche Leiharbeiter mit oder ohne Migrationshintergrund treffen und um einen schlecht bezahlten prekären Arbeitsplatz konkurrieren, dann wird das Leistungsprinzip - das bisher zur Legitimierung der nationalen Ungleichheit beitrug (Beck) - infrage gestellt. Die zu erwartenden massiven Einkommensunterschiede zwischen Leiharbeitern gleich geringer Qualifikation, jedoch unterschiedlicher Staatsangehörigkeit, gewinnen nunmehr an politischer Brisanz. Auch wenn die Leiharbeitnehmer selbst nicht in der Lage sind, als wirkungsmächtiger Akteur auf dem Arbeitsmarkt aufzutreten, da ihnen wichtige Akteurseigenschaften (formal organisiert, hierarchisch aufgebaut und zentrale Handlungsressourcen) fehlen, so trägt die derzeitige Diskussion dennoch dazu bei, dass dem Thema Leiharbeit vermehrte Aufmerksamkeit geschenkt wird.

Pikanter Weise sind es jetzt vor allem die Verleiher selbst, die Sturm laufen und trotz hoher Gewinne düstere Szenarien für den Standort Deutschland malen. Sie, die jahrelang die Spielregeln und die Definition des Leistungsprinzips in der Branche mitbestimmt haben, unterliegen $\mathrm{ab}$ Mai 2011 plötzlich ähnlichen Zwängen wie die von ihnen vermittelten Leiharbeiter.

Veränderte - und ggf. sogar erweiterte - Handlungskonstellationen tun sich aber auch für die hiesigen Gewerkschaften auf. Grenzüberschreitende Partnerschaften mit osteuropäischen Gewerkschaften könnten ein neuer Ansatzpunkt sein, sowohl den Verleihern als auch den Entleihern das Leben schwer zu machen und durch Aufklärung und Beratung die alten und neuen Leiharbeitnehmer in ihren Rechten zu stärken.

$\mathrm{Zu}$ guter Letzt wird durch die Arbeitsmarktöffnung sogar ein neuer Fokus auf die einseitige Diskussion um einen Fachkräftemangel geworfen. Jahrelang wurden in Deutschland als Folge einer unzureichenden und verschleppten Anerkennung von Berufsabschlüssen zehntausende von Migranten trotz hoher beruflicher Kompetenz in Helfertätigkeiten in die Leiharbeit abgedrängt; und die jeweils nationalen - aus deutscher Sicht globalen - Berufsqualifikationen dienten als Rechtfertigung (Beck) für ihre ungleichen Chancen auf dem deutschen Arbeitsmarkt. Dass auch andere Wege eingeschlagen werden können, zeigt die Arbeit von MigraNet, ${ }^{12}$ welche die jahrelangen politischen Versäumnisse deutlich macht.

Es steht außer Frage: Die bevorstehenden Entwicklungen auf dem Leiharbeitsmarkt zwingen zum Handeln - selbst wenn das „Worse-Case-Szenario“ nicht eintreten wird. Und sie bieten neue Handlungsoptionen. Das langfristige Ziel sollte es sein, die Leiharbeit in Deutschland wieder in den gesetzlichen Rahmen vor 2003 zurückzudrängen. Zum einen, um den Verleihern das schnelle Geldverdienen zu erschweren und den Entleihern die Möglichkeit zu nehmen, durch Leiharbeit langfristig tarifliche Vereinbarungen zu unterlaufen und Arbeitsplätze abzubauen. Zum anderen und vor allem, um die umfassenden sozialen Benachteiligungen in der Leiharbeit abzubauen, die von Einkommensdiskriminierung über betriebliche und gesellschaftliche Statusdiskriminierung bis hin zu verminderten Teilhabechancen reichen. ${ }^{13}$ Die mit dem 1. Mai 2011 eintretenden Folgen sollten deswegen Anlass sein, endlich eine Umkehr auf dem Leiharbeitsmarkt einzuleiten.

11 Beck, U. (2008): Die Neuvermessung der Ungleichheit unter den Menschen, Frankfurt, S. 16

12 MigraNet ist ein Netzwerk zur strukturellen Neuordnung der beruflichen Integration von Menschen mit Migrationshintergrund.

13 Eine erschwerte Familien- und Lebensplanung, fehlende Kreditwürdigkeit für den Erwerb von Wohneigentum und langlebige Konsumgüter und selbst die Schwierigkeit, wegen fehlender Einkommenssicherheit eine Wohnung anzumieten, zählen hier zu den typischen Beispielen - um nur einige zu nennen. 